Bundesgesundheitsbl $2019 \cdot 62: 247-254$ https://doi.org/10.1007/s00103-019-02916-y Online publiziert: 26. Februar 2019

(c) Der/die Autor(en) 2019

CrossMark

\author{
Andreas Kruse ${ }^{1}$ Gabriele Becker ${ }^{1}$ Hartmut Remmers ${ }^{2}$ Eric Schmitt ${ }^{1}$. \\ Andrea Wetzel ${ }^{3}$ \\ ${ }^{1}$ Institut für Gerontologie, Ruprecht-Karls-Universität Heidelberg, Heidelberg, Deutschland \\ ${ }^{2}$ Abteilung Pflegewissenschaft, Institut für Gesundheitsforschung und Bildung, Universität Osnabrück, \\ Osnabrück, Deutschland \\ ${ }^{3}$ AOK Baden-Württemberg, Stuttgart, Deutschland
}

\section{Selbstgestaltungs- und Präventionspotenziale hochaltriger Menschen in der stationären Langzeitversorgung}

sourcenverlusten in dieser Lebensphase wird die Erhaltung oder (möglichst weitgehende) Wiederherstellung von Selbstständigkeit ein vorrangiges Ziel gesundheitlicher Versorgung - insbesondere unter dem Aspekt der Sicherung von Möglichkeiten der Verwirklichung von Selbstverantwortung, Mitverantwortung und Teilhabe [4]. Aus diesem Grund ist es notwendig, den ärztlich-kurativen Behandlungsansatz verstärkt um präventive, rehabilitative und palliative Aspekte zu ergänzen [5-7]. Medizinische Rehabilitation hat allgemein zum Ziel, durch akute oder chronische Erkrankungen aufgetretene Funktionsverluste und -einschränkungen wieder zu beheben oder weitgehend zu kompensieren; da bei alten Menschen häufig weitere Erkrankungen vorliegen und darüber hinaus die Verletzlichkeit des Menschen im hohen Alter deutlich zunimmt, stellt die geriatrische Rehabilitation besondere Anforderungen; im Zentrum stehen dabei Einschränkungen der Mobilität, basale und instrumentelle Aktivitäten des täglichen Lebens mit der Zielsetzung, Patienten eine Rückkehr in ihr soziales Umfeld und die Aufrechterhaltung sozialer Teilhabe zu ermöglichen [8].

\section{Präventionspotenziale in der stationären Langzeitversorgung}

Auch im Pflegekontext gibt es Hinweise auf Präventionspotenziale bis ins hohe Alter. In einem Review von 92 Studien zur Prävention in der Pflege wurde moderate Evidenz für präventive Maßnahmen in der stationären Pflege konstatiert. Einzelne Wirkungsnachweise von Prävention in Pflegeheimen wurden aufgezeigt, z. B. bei ernährungsbezogenen Maßnahmen, beim Schutz durch Hüftprotektoren, bei physischem Training von bettlägerigen Patienten, bei aktivierenden Betreuungskonzepten [9].

Präventionsmaßnahmen für hochaltrige Menschen in der stationären Langzeitversorgung finden sich in vielen $\mathrm{Be}$ reichen mit sehr unterschiedlicher Zielsetzung:

1. Zahlreiche Hinweise auf die Effektivität von Prävention gibt es bei der Förderung körperlicher Aktivität. Eine Studie mit guter Evidenz konnte zeigen, dass durch Bewegungsübungen eine Verbesserung der Gehfähigkeit erreicht werden kann [10]. In einer Übersichtsarbeit, in die 14 Studien eingeschlossen waren, konnte die Wirksamkeit von Interventionen zur Förderung der körperlichen Aktivität in Pflegeheimen belegt werden. Stationär Pflegebedürftige wiesen nach Teilnahme an körperlichen Aktivi- 
täten im Vergleich zu verschiedenen Kontrollbedingungen eine signifikant höhere körperliche Funktionsfähigkeit auf. Von den Maßnahmen profitierten gerade auch körperlich und kognitiv stark beeinträchtigte Pflegebedürftige [11]. Körperliche Aktivität hat einen positiven Einfluss auf die funktionale Gesundheit, d.h. die Fähigkeit, Aktivitäten des täglichen Lebens kompetent auszuführen. Präventive Effekte sind hierbei in der Erhaltung und Verbesserung der Selbstständigkeit, der kognitiven Leistungsfähigkeit [12] sowie der gesundheitsbezogenen Lebensqualität $[13,14]$ erkennbar.

2. In der stationären Pflege besteht ein hohes Risiko der Mangel- oder Fehlernährung, die aufgrund der gesundheitlichen Folgen für die Betroffenen auch zur Zunahme des Pflegebedarfs führen kann $[15,16]$. Bei ernährungsbezogenen Maßnahmen herrscht Konsens über die vorgelegten Leitlinien $[17,18]$.

3. Maßnahmen zur Förderung oder zum Erhalt kognitiver Leistungsfähigkeit sind bei einer Demenzprävalenz von $40 \%$ in Pflegeeinrichtungen ebenfalls von hoher Bedeutung [19]. In einem Review mit zehn Interventionsstudien wurden Hinweise auf die Wirksamkeit kognitiver Trainings bei Pflegeheimbewohnern gefunden, insbesondere, wenn die kognitive Einschränkung noch nicht weit fortgeschritten war und das Trainingsprogramm individuell und über einen längeren Zeitraum durchgeführt wurde. Hier wird die insgesamt niedrige Evidenzlage der Studien betont [11].

4. Zahlreiche Präventionsmaßnahmen im Kontext stationärer Langzeiteinrichtungen zielen auf die Förderung verschiedener Aspekte psychosozialer Gesundheit im Alter. (a) Vor dem Hintergrund der hohen Prävalenzrate depressiver Störungen in Pflegeheimen (40-50\%, davon 15-20\% schwere depressive Störungen; [20]) werden präventive Maßnahmen zur Steigerung der Resilienz, Kohärenz, sinngebender Beschäftigungen und sozialer Kontakte empfohlen [19]. (b) Des Weiteren sind Maßnahmen zur Vermeidung oder Linderung von Verhaltenssymptomen bei Demenz als ein wichtiger Gegenstand psychosozialer Prävention in stationären Langzeiteinrichtungen zu nennen [21]. (c) Nur wenige Studien gibt es zur Suchtprophylaxe in Pflegeheimen, aber die überdurchschnittliche Prävalenz von Alkohol- und Substanzmissbrauch [22] weist auf dieses Präventionsfeld hin. Stärkung der Resilienz, Förderung sozialer Kontakte und Schulungen für Pflegekräfte werden hier als Präventionsmaßnahmen vorgeschlagen [23]. (d) Die Bedeutung sozialer Kontakte für die Lebensqualität, für körperliche und geistige Leistungsfähigkeit, für die Vermeidung von Gebrechlichkeit und für die Bewältigung psychischer Belastungen ist gut belegt [24, 25]. Pflegebedürftigkeit ist ein Prädiktor eingeschränkter Teilhabe; in stationären Einrichtungen ist deshalb die Vermeidung von Einsamkeit oder fehlender sozialer Integration hoch relevant [26].

5. Prävention von Gewalt in der Pflege ist ein weiteres Handlungsfeld, das Eingang in die Leitlinien zur Prävention in Pflegeeinrichtungen gefunden hat. Einrichtungsübergreifende Konzepte, die zu einer Sensibilisierung der Pflegekräfte beitragen und Handlungsleitlinien und Verantwortlichkeiten festlegen, müssen entwickelt und wissenschaftlich untersucht werden [27].

6. Ein weiterer Präventionsbereich in stationären Einrichtungen ist die Vermeidung freiheitsentziehender Maßnahmen [28]. Von diesen sind $26 \%$ der Pflegeheimbewohner betroffen, Menschen mit Demenz noch häufiger. Es konnte gezeigt werden, dass Schulungen und Motivation des Pflegepersonals, eine Anpassung der Umgebung, mehr Zuwendung und eine verstärkte nichtmedikamentöse Behandlung von demenziellen Erkrankungen zur Reduktion freiheitsentziehender Maßnahmen führen konnten [29].

\section{Selbstgestaltung als zentraler Aspekt von Prävention}

Die Prognose der Wirksamkeit einer medizinischen Behandlung hängt wesentlich von der Fähigkeit und Bereitschaft der Patienten ab, die Behandlung aktiv zu unterstützen. Als ethisch begründete, fachliche und instrumentelle Hilfestellung - und dies insbesondere für sozial benachteiligte Gruppen [30-32] - sind Angebote im Gesundheitssystem für die Umsetzung von selbstgestalteter Gesundheitsförderung und Prävention erforderlich [33]. Eine Antwort auf die erhöhte Verletzlichkeit des Menschen ist eine umfassende, sich ganz an den kognitiven und emotionalen Ressourcen des Individuums orientierende Information (Aufklärung), verbunden mit Taktund Mitgefühl sowie einer motivierenden Haltung, die nicht die objektiv gegebenen und subjektiv erlebten gesundheitlichen Grenzen des Individuums leugnet $[34,35]$. Dabei darf nicht übersehen werden, wie wichtig das mehrfach geführte Gespräch (sowohl mit den Patienten als auch mit deren Angehörigen) für die möglichst differenzierte Wahrnehmung der eigenen Veränderungs- und Entwicklungspotenziale ist. Die Umsetzung dieser Potenziale wird zusätzlich gefördert durch eine Infrastruktur, die das Individuum in seinen Bemühungen um die Aufrechterhaltung oder Wiederherstellung körperlicher und psychischer Gesundheit, wie auch allgemeiner um eine selbst- und mitverantwortliche Lebensführung unterstützt - dies sowohl im Sinne einer engen Kooperation von Hausund Fachärzten mit Vertretern anderer Gesundheitsberufe als auch im Sinne eines angemessenen (barrierefreien) $\mathrm{Zu}$ gangs zu öffentlichen Räumen, Gesundheitsinformationen und Gesundheitsangeboten $[33,36]$.

\section{Wie können Präventions- potenziale in der stationären Langzeitpflege gefördert werden? Das Beispiel ORBIT}

Den Ansatzpunkt der Prävention bilden nicht allein das Individuum und persönliche Faktoren wie Lebensstil, Alltagsgestaltung, Gesundheitsverhalten, sub- 
Bundesgesundheitsbl 2019·62:247-254 https://doi.org/10.1007/s00103-019-02916-y

(c) Der/die Autor(en) 2019

\section{A. Kruse · G. Becker · H. Remmers · E. Schmitt · A. Wetzel}

\section{Selbstgestaltungs- und Präventionspotenziale hochaltriger Menschen in der stationären Langzeitversorgung}

\section{Zusammenfassung}

Die Gesundheitsversorgung in stationären Langzeitpflegeeinrichtungen (Pflegeheimen) sollte sich nicht auf medizinisch-kurative Maßnahmen beschränken, sondern auch die Stärkung der sozialen Teilhabe, der Selbstständigkeit sowie der Selbst- und Mitverantwortung der Bewohnern einbeziehen. Prävention und Rehabilitation sollten daher noch mehr integriert werden.

In diesem Beitrag werden zunächst die verschiedenen Präventionsbereiche körperliche Aktivität, Ernährung, kognitive Leitungsfähigkeit, psychosoziale Gesundheit, Gewalt in der Pflege und unnötiger Freiheitsentzug und ihre Evidenz vorgestellt. Wesentlich für die Umsetzung und den Erfolg entsprechender Maßnahmen ist die Fähigkeit und Bereitschaft der Pflegebedürftigen, die Therapien aktiv zu unterstützen, wobei angemessene und motivierende Aufklärungsgespräche eine wichtige Rolle spielen. Im Anschluss wird auf die geriatrische Rehabilitation Bezug genommen. In der empirischen Studie ORBIT (Organisation und Rehabilitation für Bewohner im Pflegeheim zur Verbesserung der Selbstständigkeit und Teilhabe, 2013-2017) nahmen 215 Pflegebedürftige an dreimonatigen therapeutischen Interventionen mit anschließender dreimonatiger rehabilitativer Pflege teil. Im Vergleich zu einer Kontrollgruppe $(n=28)$ konnten Verbesserungen in Mobilität und Lebensqualität (Barthel-Index, Quality of Life in Alzheimer's Disease [QOL-AD]) gezeigt werden. Die Ergebnisse müssen vor dem Hintergrund eines sich im hohen Alter allgemein verschlechternden Gesundheitszustandes und sich reduzierender Fähigkeiten betrachtet werden.

Die stärkere Integration von Präventions- und Rehabilitationsangeboten in die stationäre Langzeitpflege kann die Teilhabe und Selbstständigkeit verbessern, wodurch sich nicht zuletzt die hochaltrigen Menschen in ihrer Würde geachtet, in ihrer Gesundheitskompetenz anerkannt und in ihrem Bedürfnis nach Selbstgestaltung ernst genommen fühlen.

\section{Schlüsselwörter}

Prävention im Alter · Selbstständigkeit . Selbstverantwortung · Vulnerabilität . Rehabilitative Pflege

\section{Self-design and prevention potential for older people in institutional long-term care}

\section{Abstract}

Healthcare in inpatient long-term care facilities (nursing homes) should not be limited to medical curative measures, but should also include strengthening social participation, autonomy, self-responsibility and joint responsibility of the residents. Prevention and rehabilitation should therefore be even more integrated into care concepts. This article first introduces various areas of prevention physical activity, nutrition, cognitive competence, psychosocial health, abuse, and freedom-removing measures and then discusses their evidence. Essential for the implementation and the success of such measures is the ability and willingness of people in need of care to engage actively in these therapies; here, appropriate and motivating information plays an important role.

Subsequently, geriatric rehabilitation is referred to. In the 2013-2017 empirical study Organization and Rehabilitation for Residents in the Nursing Home to Improve Independence and Participation (ORBIT), 215 people in need of care participated in threemonth therapeutic interventions, which were followed by three-months of rehabilitative care. Improvements in mobility and quality of life (Barthel index, QOL-AD) could be demonstrated compared to a control group $(n=28)$. The results have to be considered against the background of a worsening health and reduced functional capacities in old age. A stronger integration of prevention and rehabilitation services into long-term institutional care is functional for strengthening participation and independence an important condition for the residents certainty that their dignity will be respected, competence and strive for self-responsibility and self-determination.

\section{Keywords}

Prevention in old age $\cdot$ Independence $\cdot$ Selfresponsibility - Vulnerability - Rehabilitative care jektive Deutung und Bewältigung von Belastungen, Fähigkeit zur Kompensation von Einschränkungen. Vielmehr sind individuelle Bemühungen um Aufrechterhaltung oder Wiedergewinnung von Mobilität und Selbstständigkeit vor dem Hintergrund der räumlichen, der sozialen, der institutionellen und der rechtlichen Umwelt zu betrachten [32]. Bei der Entwicklung von Präventionskonzepten sind demnach auch Möglichkeiten sozialer Partizipation sowie die Zugänglichkeit sozialer, kultureller und medi- zinisch-pflegerischer Angebote für alle Menschen zu berücksichtigen [37]. Dieser Gedanke hat auch Eingang in das vom Deutschen Bundestag am 18.06.2015 verabschiedete Gesetz zur Stärkung der Gesundheitsförderung und der Prävention (Präventionsgesetz) gefunden, das unter der Zielsetzung steht, Gesundheit direkt im Lebensumfeld - sei es in der Kita, der Schule, am Arbeitsplatz oder im Pflegeheim - zu stärken.

Rehabilitative Maßnahmen können als tertiäre Prävention aufgefasst werden.
Dies gilt insbesondere in einem Kontext wie der stationären Langzeitversorgung, in dem das Ziel der Maßnahme nicht nur Verbesserung, sondern auch der Erhalt von Kompetenzen, die Verzögerung des Abbaus von Fähigkeiten oder die Erhaltung der Lebensqualität ist. Bei Menschen mit Pflegebedarf sind Prävention und Rehabilitation kaum $\mathrm{zu}$ trennen. So ist beispielsweise das Konzept der aktivierenden Pflege eher der Prävention und Gesundheitsförderung zuzuordnen, enthält aber auch 
rehabilitative Elemente [38]. Nur wenn Prävention und Rehabilitation miteinander verzahnt werden, ist eine optimale Versorgung nahe an den Bedarfen der Pflegebedürftigen möglich [39, 40]. Die eingesetzten therapeutischen Konzepte unterscheiden sich weniger in der Zuordnung zu Prävention oder Rehabilitation, sondern müssen vor allem auf Menschen im hohen Alter zugeschnitten sein, indem bei ihrer Umsetzung die individuellen Einbußen und Bedarfe genauso wie die noch vorhandenen Ressourcen berücksichtigt werden [41]. Das Institut für Gerontologie und die AOK Baden-Württemberg haben im Projekt ORBIT (Organisation der Rehabilitation für Bewohner im Pflegeheim zur Verbesserung der Selbstständigkeit und Teilhabe, Laufzeit 2013 bis 2017) ein Konzept zur Umsetzung rehabilitativer Pflege entwickelt. Das Angebot richtete sich an Pflegeheimbewohner mit Rehabilitationsbedarf und -potenzial.

\section{Ziele der Studie}

Zielsetzung des Projekts war die Integration von rehabilitativen Maßnahmen in die Pflege. Die Fragestellungen waren, welches Entwicklungspotenzial bei Bewohnern von Altenheimen vorhanden ist und wie es durch eine rehabilitative Pflege gefördert werden kann. Zielgrößen waren dabei in erster Linie die Verbesserung der Mobilität und der Lebensqualität. Die Umsetzung der rehabilitativen Pflege basierte auf der fachlichen Kompetenz der Pflegefachkräfte und der Therapeuten. Eine weitere Zielsetzung des Projekts war daher die Förderung der interdisziplinären Zusammenarbeit der beiden Berufsgruppen.

\section{Methode}

Das Konzept von ORBIT bestand in einem mehrstufigen Verfahren. Nach der Identifikation des Interventionsbedarfs im Pflegekontext erfolgte ein durch eine Pflegefachkraft ausführlich begründeter Vorschlag für eine Physio- oder Ergotherapie oder eine Logopädie, der die Formulierung des rehabilitativen Bedarfs und der individuellen Potenziale sowie einen Therapievorschlag umfasste. Es gab keine Ausschlusskriterien, auch Personen mit fortgeschrittener Demenz und/ oder schwerer Pflegebedürftigkeit konnten teilnehmen. Der Therapievorschlag und die ausreichende Belastbarkeit für die Therapie wurden vom Hausarzt des Pflegeheimbewohners geprüft und das entsprechende Heilmittel verordnet. Die intensive Therapie erfolgte zweimal wöchentlich über einen Zeitraum von drei Monaten. Nach der Therapie wurden im schriftlichen Therapiebericht Empfehlungen bestimmter Therapieelemente gegeben, die weitere drei Monate durch Pflegefachkräfte weitergeführt werden sollten. Die Empfehlungen bestanden je nach individuellem Bedarf in Gehoder Bewegungsübungen, in passiver Bewegung, Lagerung oder in Anleitungen bzw. Motivierung zu empfohlenen Übungen. Zu Beginn der begleitenden Datenerhebung (1. Messzeitpunkt (MZP), T1) wurde ein ausführliches geriatrisches Assessment durchgeführt, das nach der Therapie (2. MZP, T2) sowie nach weiteren drei Monaten rehabilitativer Pflege (3. MZP, T3) wiederholt wurde. Das Assessment erfasste neben soziodemografischen Merkmalen Gesundheitsstatus, Funktionalität, kognitive Leistungsfähigkeit, Depressivität und Lebensqualität. Zur Beschreibung des Gesundheitsstatus wurde die Zahl schwerer Erkrankungen mit Diagnosen erfasst und der Morbiditätsindex aus dem Cumulative Illness Rating Scale for Geriatrics (CIRS-G; [42]) bestimmt, der die relevanten Erkrankungen in 13 Organsystemen zusammenfasst. Der Grad der Selbstständigkeit wurde mit dem Barthel-Index [43] und die kognitive Leistungsfähigkeit mit dem Mini-mental State Test (MMST; [44]) eingeschätzt. Für die Bestimmung der Lebensqualität wurde der Quality of Life in Alzheimer's Disease (QOL-AD; [45]) eingesetzt, ein Fragebogen zur Selbst- bzw. Fremdeinschätzung der Lebensqualität, der auch bei Menschen mit Demenz eingesetzt werden kann. Neben quantitativen Daten wurden auch qualitative Daten erhoben. In leitfadengestützten Interviews wurden jeweils die Pflegefachkräfte und Pflegeheimbewohner nach ihrer Einschätzung des Therapieerfolges befragt. In Experteninterviews mit Therapeu- ten, Wohnbereichs-, Pflegedienst- und Heimleitern wurde nachgefragt, wie die Kooperation von Therapeuten und Pflegekräften verlief und in welchem Umfang die Empfehlungen der Therapeuten in der rehabilitativen Pflege umgesetzt werden konnten.

\section{Stichprobe}

Am Projekt waren 13 Pflegeheime, 59 Hausärzte und 39 therapeutische Einrichtungen beteiligt. Insgesamt wurden 215 Pflegeheimbewohner mit Rehabilitationsbedarf in die Studie aufgenommen. - Tab. 1 gibt einen Überblick über dieStudienteilnehmer in ausgewählten Merkmalen. Deutlich werden dabei die hohe Prävalenz körperlicher und psychischer Erkrankungen, darunter in erster Linie Erkrankungen des Bewegungsapparates und mittlere und schwere Demenz, der ausgeprägte Pflegebedarf und die hohe Zahl der Krankenhausaufenthalte der vergangenen 12 Monate.

Bei 139 Teilnehmern konnte eine komplette Datenreihe mit drei Messzeitpunkten erhoben werden. 48 Teilnehmer schieden vor dem 3. Messzeitpunkt wegen Erkrankungen, Versterben oder Verlegung aus. Gleichzeitig wurde eine Kontrollgruppe von 28 Personen aufgebaut, die ihre Therapie später oder gar nicht erhielten. $\bullet$ Tab. 2 zeigt die durchschnittliche Zahl schwerer Erkrankungen, Morbiditätsindex, Barthel-Index und MMST in der Interventionsstichprobe $(n=139)$ und der Kontrollgruppe $(n=28)$. Die Werte unterschieden sich nicht signifikant.

\section{Ergebnisse}

\section{Entwicklungen in der Funktio- nalität mit und ohne Therapie (T1-T2)}

$76 \%$ der Teilnehmer erhielten eine Physiotherapie. Ergotherapien und Logopädie wurden deutlich seltener verordnet. Die funktionellen Fähigkeiten der Studienteilnehmer mit Interventionsbedarf $(n=139)$ verbesserten sich während der Therapie (Zugewinn im Barthel-Index $+1,4$ Punkte), dies war auch bei schwerem Pflegebedarf (Barthel-Index zu T1 $\leq 20$ Punkte) zu beob- 
Tab. 1 Stichprobenbeschreibung bei Aufnahme (T1) in die Studie „Organisation und Rehabilitation für Bewohner im Pflegeheim zur Verbesserung der Selbstständigkeit und Teilhabe" (ORBIT) Stichprobenmerkmale

Anteil weiblicher Heimbewohner

Durchschnittsalter

Pflegestufen

0

I

II

III

Krankenhausaufenthalte im letzten Jahr

Schwere Erkrankungen des Bewegungsapparates

Schwere psychische Erkrankungen

\begin{tabular}{|l|l|l|l|}
\hline & Tab. 2 & Vergleich der Interventions- und Kontrollgruppe bei Aufnahme in die Studie (T1) \\
& $\begin{array}{l}\text { Interventionsgruppe } \\
\boldsymbol{n}=\mathbf{1 3 9}\end{array}$ & $\begin{array}{l}\text { Kontrollgruppe } \\
\boldsymbol{n}=\mathbf{2 8}\end{array}$ & $\begin{array}{l}\text { t-Test, } \\
\text { Signifikanz }\end{array}$ \\
\hline Schwere Erkrankungen & 2,63 & 2,61 & 0,08 n.s. \\
\hline Morbiditätsindex & 17,1 & 17,6 & $-0,47$ n.s. \\
\hline Barthel-Index & 39,1 & 37,9 & 0,24 n.s. \\
\hline Mini-Mental-Status-Test & 19,8 & 18,0 & 0,99 n.s.
\end{tabular}

achten ( $n=44$, Zugewinn $+2,1$ Punkte). Der Effekt der Intervention wird durch den Vergleich mit der Kontrollgruppe deutlich. Die Entwicklung im BarthelIndex war in der Interventionsgruppe signifikant besser als in der Kontrollgruppe $(n=28)$, dort verschlechterte sich der Mittelwert (-2,7 Punkte). Der Anteil der Personen, die sich im Barthel-Index verbessern konnten, betrug in der Interventionsgruppe $36 \%$ vs. $7 \%$ in der Kontrollgruppe. Gleichzeitig wurde deutlich, dass ohne rehabilitative Maßnahmen Selbstständigkeitseinbußen ausgeprägter waren. Im Beobachtungszeitraum von 3-4 Monaten verschlechterten sich im Barthel-Index in der Kontrollgruppe ohne Intervention $36 \%$ der Personen vs. $22 \%$ in der Interventionsgruppe. Bei $70 \%$ der Stichprobe waren Verbesserungen Interventionsziel, bei $30 \%$ war zumindest ein Erhalt des funktionellen Status angestrebt worden.

\section{Entwicklungen in Abhängigkeit von der Umsetzung von Therapieempfehlungen (T2-T3)}

Bei $56 \%$ der Interventionsgruppe konnten die Therapieempfehlungen vom Pflegefachpersonal umgesetzt werden. Gründe für die nur teilweise oder nicht gelungene Umsetzung waren die mangelnde Belastbarkeit (z. B. bei komplizierten
Verläufen) und die fehlende Motivation der Pflegeheimbewohner, aber auch ein Mangel an zeitlichen Ressourcen der Pflegefachkräfte. Teilte man die Stichprobe nach der Umsetzbarkeit der Therapeutenempfehlungen in zwei Gruppen, ließ sich ein signifikanter Unterschied in der funktionellen Entwicklung nachweisen. Bei 73 Teilnehmern führte die rehabilitative Pflege zwischen T2 und T3 $\mathrm{zu}$ einer weiteren Verbesserung von 1,4 Punkten im Barthel-Index, während in der Gruppe von 57 Teilnehmern, bei denen die Empfehlungen nur teilweise oder gar nicht umgesetzt werden konnten, die Barthel-Werte wieder um durchschnittlich 5,1 Punkte schlechter wurden.

\section{Entwicklungen in Abhängigkeit von Komplikationen im Gesamtverlauf}

Die Therapie und die nachfolgende rehabilitative Pflege waren erfolgreicher, wenn der Verlauf ungestört von Komplikationen war. Dies war in $43 \%$ der Verläufe $(n=56)$ der Fall. Die deutlichen Verbesserungen im Barthel-Index, die in der Therapie erreicht werden konnten, konnten unter der rehabilitativen Pflege erhalten werden. Der hohe Anteil komplizierter Verläufe $(n=83)$ zeigte das Maß der Vulnerabilität bei den Teilnehmern der Untersuchung. Bei den Komplikationen handelte es sich meist um Stürze (15\%), Infektionen der Atemwege (12,5\%) oder Depressivität $(10 \%)$. In dieser Gruppe entwickelten sich die funktionellen Fähigkeiten negativ, auch deswegen, weil die Umsetzung der Intervention teilweise eingeschränkt war. Den durchschnittlichen Barthel-Index bei Verläufen ohne und mit Komplikationen zeigt die - Tab. 3.

\section{Therapieerfolg und Lebensqualität}

Die Niveaus der Durchschnittswerte im QOL-AD bei der Selbsteinschätzung ( $n=74 ; 31,2$ Punkte, Wertebereich 13-65 Punkte) und der Fremdeinschätzung $(n=65 ; 23,7)$ unterschieden sich deutlich, da die Personen, die nicht selbst Auskunft geben konnten, auch funktionell in einem deutlich schlechteren Zustand waren. Während der Intervention $(\Delta \mathrm{T} 1 \mathrm{~T} 2)$ wurden mit einem Zuwachs um 1,95 bzw. 1,63 Punkte signifikante Verbesserungen bei der selbstund der fremdeingeschätzten Lebensqualität erreicht. Im Intervall zwischen T2 und T3 verschlechterten sich die Durchschnittswerte wieder. Bei den von den Teilnehmern selbst vorgenommenen Einschätzungen ergaben sich in diesem Zeitraum mit einer Verminderung um 1,3 Punkte sogar signifikant schlechtere Werte. Eine besondere Stellung nahm die Gruppe der schwer pflegebedürftigen Heimbewohner $(n=44)$ ein. Bei ihnen konnte eine fortgesetzte Verbesserung der fremdeingeschätzten Lebensqualität über den ganzen Beobachtungszeitraum festgestellt werden $(\Delta \mathrm{T} 1 \mathrm{~T} 2=+2,3$ Punkte und $\Delta \mathrm{T} 2 \mathrm{~T} 3=0,2$ Punkte). Damit hatten diese Personen eine signifikante Verbesserung der Lebensqualität am Ende der Studie $(\Delta \mathrm{T} 1 \mathrm{~T} 3=2,5$ Punkte, Wilcoxon-Test U-Wert $\left.-2,5^{*}\right)$.

Es bestand ein signifikanter Zusammenhang zwischen einer Verbesserung der Lebensqualität und einer gleichzeitigen Verbesserung der Selbstständigkeit, gemessen mit dem BarthelIndex (•Tab. 4). Der Zusammenhang ist deutlicher bei der selbsteingeschätzten Lebensqualität und betrifft sowohl den Zeitabschnitt während der therapeutischen Intervention $(\Delta$ T1T2) als auch die Phase der rehabilitativen Pflege $(\Delta$ T2T3). 
Tab. 3 Barthel-Index bei Therapieverlauf ohne Komplikationen $(n=56)$ und mit Komplikationen $(n=83)$ über 3 Messzeitpunkte T1, T2, T3

\begin{tabular}{|l|l|l|l|}
\hline Verlaufsparameter & \multicolumn{3}{l}{ Mittelwerte (SD, Signifikanzniveau vs. T1) } \\
& T1 & T2 & T3 \\
\hline Barthel-Index (ohne Komplikationen, $n=56)$ & $38,8(25,1)$ & $43,1(27,5 ; * * *)$ & $43,3(27,8 ; * * *)$ \\
\hline Barthel-Index (mit Komplikationen, $n=83)$ & $39,3(24,6)$ & $38,7(23,1 ;$ n. s.) & $36,2\left(23,5 ;{ }^{*}\right)$ \\
\hline${ }^{*} p<0.05 ;{ }^{* * *} p<0.005 ;$ n. s. nicht signifikant & & & \\
\hline
\end{tabular}

Tab. 4 Korrelationen zwischen Verbesserungen der Lebensqualität ( $\triangle \mathrm{QOL}-\mathrm{AD}$, selbst- und fremdeingeschätzt) und der Selbstständigkeit ( $\triangle$ Barthel-Index) in verschiedenen Messintervallen

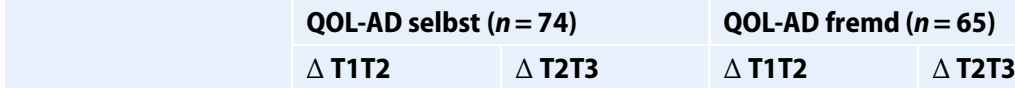

\begin{tabular}{l|llll}
\hline $\begin{array}{l}\text { Barthel-Index } \\
\Delta \mathrm{T} 1 \mathrm{~T} 2\end{array}$ & $0,333^{* * *}$ & 0,010 n.s. & $0,287^{*}$ & 0,076 n.s. \\
$\Delta \mathrm{T} 2 \mathrm{~T} 3$ & 0,105 n.s. & $0,416^{* * *}$ & $-0,075$ n.s. & $0,316^{*}$
\end{tabular}

${ }^{*} p<0.05 ;{ }^{* * *} p<0.005 ;$ n. s. nicht signifikant

\section{Diskussion}

Die Studie ORBIT deckte zusätzlichen Rehabilitations- und Präventionsbedarf in Pflegeeinrichtungen auf. In erster Linie sollten mit den Maßnahmen Verbesserungen in der Mobilität und Selbstständigkeit erreicht werden. Bei der Gewinnung von Studienteilnehmern wurde den Vorschlägen der Pflegekräfte gefolgt, die neben dem Bedarf nach rehabilitativen Maßnahmen bei den Heimbewohnern auch die für das Erreichen des Therapiezieles erforderlichen Potenziale aufdeckten und beschrieben. Das bei Pflegeheimbewohnern vorhandene Potenzial konnte durch die Entwicklungen unter der Intervention bestätigt werden, insbesondere durch den Vergleich mit der Kontrollgruppe. Auch andere Studien zu rehabilitativen Maßnahmen in der Pflege haben positive Effekte auf die Funktionalität gezeigt. Im Vergleich mit Kontrollgruppen lagen sie zum Teil in Verbesserungen [46] oder in einem geringeren Abbau [47]. Die Therapien können auch als präventive Maßnahmen zur Verminderung funktionalen Abbaus aufgefasst werden [48]. Der Vergleich mit der Kontrollgruppe gibt Hinweise, wie Entwicklungen verlaufen, wenn bei Interventionsbedarf Therapien ausbleiben. Angesichts der häufig beobachteten Verluste lässt sich nachvollziehen, dass die individuell angemessenen Therapieziele in der Interventionsgruppe nicht ausschließlich in Verbesserungen der Fähigkeiten, son- dern in $30 \%$ der Fälle in einem Erhalt des Anfangsstatus lagen.

Der Erfolg der rehabilitativen Pflege war abhängig von einer gelungenen Integration rehabilitativer Elemente in die Pflege. Für diese Integration war eine gute Kommunikation und Zusammenarbeit zwischen den Pflegekräften und Therapeuten Voraussetzung. Es war vorteilhaft, wenn die Übungen klar beschrieben und leicht umsetzbar waren. Die Kommunikation zwischen den beiden Berufsgruppen war durch unterschiedliche fachliche Konzepte geprägt. Die Pflegefachkräfte wünschten sich ein besseres gerontologisches Fachwissen bei den Therapeuten, diese fanden besseres Wissen der Pflegefachkräfte über Therapiekonzepte, z.B. in der Physiotherapie, hilfreich. Die interdisziplinäre Zusammenarbeit muss daher bereits in der Ausbildung gefördert und in der Versorgungspraxis strukturiert werden. Andere Kontextfaktoren, die Einfluss auf die Umsetzung der Therapeutenempfehlungen hatten, waren die Motivation und Kooperation der Studienteilnehmer bei den täglichen Übungen, aber auch Erkrankungen oder Schmerzzustände sorgten für Diskontinuität bei der Umsetzung. Ein weiterer Grund waren die eingeschränkten zeitlichen Möglichkeiten der Pflegefachkräfte, die Übungen regelmäßig durchführen zu können. So wurde Personalmangel als Grund dafür angegeben, dass in einigen Fällen die Umsetzung der rehabilitativen Pflege nicht möglich war.
Die Ergebnisse zeigen, dass Interventionserfolge eng mit Verbesserung der Lebensqualität korrelieren. Bei einem Funktionsverlust nach Beendigung der Therapie nahm entsprechend die Lebensqualität wieder ab. Die Entwicklungen der Lebensqualität und funktionellen Fähigkeiten bei den Personen mit hohem Pflegebedarf weisen auf anhaltende positive Effekte von Förderung und Zuwendung im Rahmen einer rehabilitativen Pflege bei Menschen mit ausgeprägter Vulnerabilität hin. Bei Menschen mit Demenz konnten bereits durch rehabilitative Pflege positive Effekte auf funktionelle und kognitive Fähigkeiten gezeigt werden [49]. Die hohe Prävalenz von Komplikationen ist ebenfalls als Ausdruck der hohen Vulnerabilität von Pflegeheimbewohnern anzusehen. Bei komplikationslosem Verlauf konnten Potenziale besser ausgeschöpft und bessere Ergebnisse unter der Therapie erreicht werden und bis zum dritten MZP gelang es in der Regel, das Ergebnis $\mathrm{zu}$ stabilisieren. Der Befund weist auf die Bedeutung der Prävention vermeidbarer Komplikationen, wie z. B. der Sturzprophylaxe, hin.

\section{Ausblick}

Die deutlich stärkere Integration von Präventions- und Rehabilitationsmaßnahmen (Letztere auch verstanden im Sinne der tertiären Prävention) in die Betreuungsangebote stationärer Langzeitpflegeeinrichtungen ist vor dem Hintergrund der hier berichteten Arbeiten als eine zentrale Versorgungsaufgabe $\mathrm{zu}$ werten. Die erhöhte Verletzlichkeit von Bewohnern darf nicht zu dem Fehlschluss nicht mehr gegebener Veränderungspotenziale führen. Zudem darf nicht der Fehler begangen werden, aufgrund der einseitigen Betonung von Verletzlichkeit die seelisch-geistigen Wachstumspotenziale zu übersehen, die Menschen in die Lage versetzen, auch bei starken gesundheitlichen und funktionellen Einbußen ihre Fähigkeiten zur Selbst- und Weltgestaltung zu bewahren. Die Umsetzung dieser Fähigkeiten profitiert von der stärkeren Integration der Präventionsund Rehabilitationsangebote nicht nur deswegen, weil prinzipiell vorhandene 
Plastizität genutzt wird, sondern auch deswegen, weil sich Bewohner in ihrer Würde geachtet, in ihrer Gesundheitskompetenz anerkannt und in ihrem Bedürfnis nach Selbstgestaltung ernst genommen fühlen.

\section{Korrespondenzadresse}

\section{Andreas Kruse}

Institut für Gerontologie, Ruprecht-Karls-

Universität Heidelberg

Bergheimer Str. 20, 69115 Heidelberg,

Deutschland

Andreas.kruse@gero.uni-heidelberg.de

\section{Einhaltung ethischer Richtlinien}

Interessenkonflikt. A. Kruse, G. Becker, H. Remmers, E. Schmitt und A. Wetzel geben an, dass kein Interessenkonflikt besteht.

Die Autoren haben sich bei der Planung und Durchführung der Studie an den ethischen Richtlinien der 13 beteiligten Einrichtungen sowie der AOK Baden-Württemberg orientiert. In allen Einrichtungen wurden regelmäßig ethische Fallkonferenzen durchgeführt. Dieses Verfahren wurde gewählt, weil in dieser Studie die Entscheidung über Verordnungen und Therapien allein bei Ärzten und Therapeuten lag.

Open Access. Dieser Artikel wird unter der Creative Commons Namensnennung 4.0 International Lizenz (http://creativecommons.org/licenses/by/4.0/deed. de) veröffentlicht, welche die Nutzung, Vervielfältigung, Bearbeitung, Verbreitung und Wiedergabe in jeglichem Medium und Format erlaubt, sofern Sie den/die ursprünglichen Autor(en) und die Quelle ordnungsgemäßnennen, einen Linkzur Creative Commons Lizenz beifügen und angeben, ob Änderungen vorgenommen wurden.

\section{Literatur}

1. Deutscher Bundestag (2006) Potenziale des Alters in Wirtschaft und Gesellschaft. Der Beitrag älterer Menschen zum Zusammenhalt der Generationen. Fünfter Altenbericht der Bundesregierung Bundestagsdrucksache 16/2190. Deutscher Bundestag, Berlin

2. Carstensen LL, Lang FR (2007) Sozioemotionale Selektivität über die Lebensspanne: Grundlagen und empirische Befunde. In: Brandtstädter J, Lindenberger U (Hrsg) Entwicklungspsychologie der Lebensspanne. Kohlhammer, Stuttgart, S 389-412

3. Kruse A (2017) Lebensphase hohes Alter Verletzlichkeit und Reife. Springer, Heidelberg

4. Deutscher Bundestag (2016) Siebter Bericht zur Lage der älteren Generation in der Bundesrepublik Deutschland. Sorge und Mitverantwortung in der Kommune - Aufbau und Sicherung zukunftsfähiger Gemeinschaften. Bundestagsdrucksache 18/10210. Deutscher Bundestag, Berlin

5. v Renteln-Kruse W, Anders J, Dapp U, MeierBaumgartner HP (2003) Präventive Hausbesuche durch eine speziell fortgebildete Pflegefachkraft bei 60-jährigen und älteren Personen in Hamburg ZGerontol Geriatr 36:378-391

6. v Renteln-Kruse W, Minder CE (2016) It is time to detect preclinical signs of incipient frailty. J Am Geriatr Soc 64:2166-2167

7. Remmers H (2010) Der Beitrag der Palliativpflege zur Lebensqualität demenzkranker Menschen. In: Kruse A (Hrsg) Lebensqualität bei Demenz? Akademische Verlagsgesellschaft, Heidelberg, S117-137

8. Gogol M (2014) Rehabilitation. In: Pantel J, Schröder J, Bollheimer C (Hrsg) Praxishandbuch Altersmedizin. Geriatrie - Gerontopsychiatrie Gerontologie. Kohlhammer, Stuttgart, S694-700

9. Görres S, Schmitt S, Neubert L, Zimmermann M, Stolle C (2013) Prävention in der Pflege. Maßnahmen und ihre Wirksamkeit. Abschlussbericht für das ZQP

10. Crocker T, Forster A, Young J et al (2013) Physical rehabilitation for older people in longterm care. Cochrane Database Syst Rev. https://doi.org/10. 1002/14651858.CD004294.pub3

11. Wöhl C, Richter S, Blättner B (2017) Kognitive Interventionen in Pflegeheimen. Systematische Übersicht der präventiven Wirksamkeit auf die kognitive Leistungsfähigkeit von Pflegebedürftigen. Z Gerontol Geriatr. https://doi.org/10.1007/ s00391-017-1330-3

12. Littbrand H, Stenvall M, Rosendahl E (2011) Applicability and effects of physical exercise on physical and cognitive functions and activities of daily living among people with dementia. A systematic review. Am J Phys Med Rehabil 90:495-518

13. v Malderen L, Mets T, Gorus E (2013) Interventions to enhance the quality of life of older people in residential long-term care: a systematic review. Ageing Res Rev 12:141-150

14. Remmers H (2016) Zur Relevanz des Körpers im Kontext pflegerischen Handelns. In: Uschok A (Hrsg) Körperbild - und Körperbildstörungen. Handbuch für Pflege- und Gesundheitsberufe. Hogrefe, Bern, S 25-43

15. Bauer J, Sieber C (2008) Sarcopenia and frailty - a clinician's controversial point of view. Exp Gerontol 43:674-678

16. Sieber C (2014) Frailty. In: Pantel J, Schröder J, Bollheimer C (Hrsg) Praxishandbuch Altersmedizin. Geriatrie-Gerontopsychiatrie-Gerontologie. Kohlhammer, Stuttgart, S84-93

17. VolkertD, Bauer JM, Frühwald Tetal (2013) Leitlinie der Deutschen Gesellschaft für Ernährungsmedizin (DGEM) in Zusammenarbeit mit der GESKES, der AKE und der DGG. Klinische Ernährung in der Geriatrie. Aktuel Ernahrungsmed 38:e1-e48

18. Deutsches Netzwerk für Qualitätsentwicklung in der Pflege (DNQP) (2010) Expertenstandard „Ernährungsmanagement in derPflege"

19. GKV-Spitzenverband (2018) Leitfaden für Prävention in stationären Pflegeeinrichtungen nach $\S 5$ SGB XI.GKV-Spitzenverband, Berlin

20. Weyerer S, Bickel H (2007) Epidemiologie psychischer Erkrankungen im höheren Lebensalter. Kohlhammer, Stuttgart

21. AWMF (2016) Leitlinien Demenz Entwicklungsstufe S3. AWMF-Register-Nummer:038-01

22. Weyerer S, Schäufele M, Hendlmeier I (2006) Alkoholmissbrauch und -abhängigkeit bei Bewohnern und Bewohnerinnen in Altenpflegeheimen. Repräsentative Ergebnisse aus der Stadt Mannheim ZGerontopsychol Gerontopsychiatr 19:229-235

23. Wolter DK (2016) Schmerzen und Schmerzmittelabhängigkeit im Alter: Die gerontopsychiatrische Perspektive. Kohlhammer, Stuttgart
24. Bunt S, Steverink N, Olrhof J, van der Schans CP (2017) Social frailty in older adults: a scoping review. Eur J Ageing 14:323-334

25. Gerstorf D, Hoppmann CA, Löckenhoff CE et al (2016) Terminal decline in well-being: the role of social orientation. Psychol Aging 31:149-165

26. Forsman AK, Schierenbeck I, Wahlbeck K (2011) Psychosocial interventions for the prevention of depression in older adults: systematic review and meta-analysis. J Aging Health 23:387-416

27. Meyer G, Abraham J (2013) Gewaltprävention in der Pflege. Übersichtsarbeit zu Voraussetzungen und wirksamen Maßnahmen zur Vermeidung von Gewalt in der Pflege. Abschlussbericht für das Zentrum Qualität in der Pflege. https://www. zqp.de/portfolio/projekte-studie-wirksamkeitgewaltpraevention/.Zugegriffen: 15. Sept. 2018

28. Deutscher Ethikrat (2018) Wohltätiger Zwang. Stellungnahme. Deutscher Ethikrat, Berlin

29. Meyer G, Möhler R, Köpke S (2016) Freiheitsentziehende Maßnahmen in der Altenpflege. Beitrag der Wissenschaft zur Qualitätssicherung. Arztebl Sachsen 2:70-73

30. Kruse A, SchmittE (2015) Shared responsibility and civic engagement in very old age. Res Hum Dev 12:133-148

31. Naegele G (Hrsg) (2010) Soziale Lebenslaufpolitik. SpringerVS, Wiesbaden

32. Naegele G (2016) 25 Jahre sozialgerontologische Forschung in Dortmund. In: Naegele G, Olbermann E, Kuhlmann A (Hrsg) Teilhabe im Alter gestalten. SpringerVS, Wiesbaden, S13-44

33. Kümpers S, Zander M (2012) Der Autonomiebegriff im Kontext von Hilfe- und Pflegebedürftigkeit und sozialer Benachteiligung. In: Kümpers S, Heusinger I (Hrsg) Autonomie trotz Armut und Pflegebedarf? Altern unter Bedingungen von Marginalisierung. Huber, Bern, S21-38

34. Frühwald T(2012) Ethik in der Geriatrie. Z Gerontol Geriatr 45:545-557

35. Schweda M (2014) „Ein Jegliches hat seine Zeit”. Altern und die Ethik des Lebensverlaufs. Z Prakt Philos 1:185-232

36. Klein J, KnesebeckO (2016) Soziale Unterschiede in der ambulanten und stationären Versorgung. Ein Überblick über aktuelle Befunde aus Deutschland. Bundesgesundheitsblatt Gesundheitsforschung Gesundheitsschutz 59:238-244

37. Kruse A(2002) Gesund Altern. Stand derPrävention und Entwicklung ergänzender Präventionsstrategien. Nomos, Baden-Baden

38. Dangel B, Korporal J (2003) Kann Pflege im Rahmen der Pflegeversicherung Grundlage eines spezifischen pflegerischen Ansatzes der Rehabilitation sein? Z Gerontol Geriatr 36:50-62

39. Walter U (2008) Möglichkeiten der Gesundheitsförderung und Prävention im Alter. In: Kuhlmey A, SchaefferD(Hrsg) Alter, Gesundheitund Krankheit. Huber, Bern, S245-262

40. Schaeffer D, Moers M (2011) Bewältigung chronischer Krankheiten - Herausforderungen für die Pflege. In: Schaeffer D, Wingenfeld K (Hrsg) Handbuch Pflegewissenschaft. Juventa, Weinheim, S329-363

41. Remmers H, Dütthorn N, Garthaus M (2013) Neue Pflege - Pflegerische Betreuung im Kontext rehabilitativer, präventiver und palliativer Pflegepotenziale. In: Hoppe HP (Hrsg) Pflege im Umbruch. Schlütersche, Hannover, S45-69

42. NosperM (2003) Manual CIRS-G. Cumulative Illness Rating Scale. Skala zur kumulierten Bewertung von Erkrankungen. Medizinischer Dienst der Krankenversicherung Rheinland Pfalz, Alzey 


\section{Leitthema}

43. Mahoney Fl, Barthel DW (1965) Functional evaluation: the Barthel index. Md Med J 14:56-61

44. Folstein M, Folstein S, Mc Mugh P (1975) „Minimental state": a practical method for grading the cognitive state of patients for the clinician. JPsychiatr Res 12:189-198

45. Logsdon R, Gibbons L, McCurry S, Teri L (1999) Quality of life in Alzheimer's disease: patient and caregivers report. J Ment Health Aging 5:21-32

46. Resnick B, Gruber-Baldini AL, Zimmerman S et al (2009) Nursing home resident outcomes from the rescare intervention. J Am Geriatr Soc 57:1156-1165. https://doi.org/10.1111/j.15325415.2009.02327.x

47. Kerse N, Peri K, Robinson E et al (2008) Does a functional activity programme improve function, quality of life, and falls for residents in long term care? Cluster randomised controlled trial. BMJ 337:a1445

48. Dechamps A, Diolez P, Thiaudière E et al (2010) Effects of exercise programs to prevent decline in health-related quality of life in highly deconditioned institutionalized elderly persons. Arch Intern Med 170:162-169

49. Korczak D, Habermann C, Braz S (2013) The effectiveness of occupational therapy for persons with moderate and severe dementia. GMS Health Technol Assess. https://doi.org/10.3205/ hta000115 